\title{
Pressão intraocular antes e após a facoemulsificação em olhos normais
}

\author{
Intraocular pressure before and after \\ phacoemulsification in normal eyes
}

Nikias Alves da Silva1', Felício Aristóteles da Silva²

\section{Resumo}

Objetivo: Avaliar comparativamente a pressão intraocular (PIO) antes e após 1 dia, 3 dias, 1 semana, 1 mês, 3 meses, 6 meses e 1 ano, em olhos normais submetidos à facoemulsificação. Métodos: Análise retrospectiva da pressão intraocular (medida pelo tonômetro de aplanação de Goldmann) de 221 olhos normais de 133 pacientes submetidos à facoemulsificação sem intercorrências. A PIO foi avaliada antes e após a facoemulsificação (1dia, 3 dias, 1 semana, 1 mês, 3 meses, 6 meses e 1 ano), e os valores encontrados nestes períodos foram submetidos a uma análise comparativa. Resultados: A PIO média foi de $16,06 \pm 2,69 \mathrm{mmHg}$ no pré-operatório; $18,76 \pm 5,96 \mathrm{mmHg}$ após 1 dia;

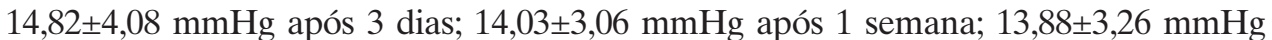

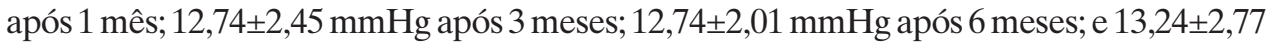
mmHg após 1 ano. A PIO aumentou 18,9\% após 1 dia; diminuiu $6,9 \%$ após 3 dias; $12,3 \%$ após 1 semana; $12,5 \%$ após 1 mês; $19,3 \%$ após 3 meses; 20,1\% após 6 meses; e 19,9\% após 1 ano. Conclusão: A cirurgia de facoemulsificação sem intercorrências leva a uma redução significativa da PIO em olhos normais. A diminuição da PIO é mais acentuada naqueles pacientes que apresentam PIO mais alta no pré-operatório.

Descritores: Pressão intraocular; Tonometria ocular; Facoemulsificação; Implante de lente intraocular; Cápsula do cristalino

\footnotetext{
'Preceptor da Unidade de Catarata e Glaucoma da Clínica de Olhos da Santa Casa de Misericórdia de Belo Horizonte, Médico Oftalmologista do Centro de Oftalmologia Avançada (COA); Pós-graduando (Doutorado) do Hospital São Geraldo - Hospital das Clínicas/UFMG - Belo Horizonte(MG) - Brasil;

2Professor Voluntário da Clínica de Olhos da Santa Casa de Misericórdia de Belo Horizonte; Associado do Centro de Oftalmologia Avançada, Belo Horizonte (MG) - Brasil.

Trabalho realizado no Centro de Oftalmologia Avançada - COA - Belo Horizonte (MG), Brasil
} 


\section{INTRODUÇÃO}

$\Delta$ cirurgia de catarata tem sido cada vez mais frequente, o que se deve ao aumento da expectativa de vida da população, a uma intervenção cirúrgica mais precoce e ao aprimoramento da técnica operatória, mormente com o advento da facoemulsificação.

Mudanças no diâmetro da câmara anterior $(\mathrm{ACD})^{(1-6)} \mathrm{e}$ na pressão intraocular (PIO) $)^{(4-10)}$ são esperadas após a facectomia e implante da lente intraocular. Aumento $^{(1,2,4-6)}$ ou diminuição ${ }^{(3)}$ do ACD após a facectomia já foram previamente demonstrados, assim como uma redução da PIO ${ }^{(4-7)}$. Aumento do ACD e da amplitude do seio camerular foram mecanismos propostos no intuito de se explicar a diminuição da $\mathrm{PIO}^{(4)}$.

O objetivo deste estudo foi investigar e comparar as medidas da PIO antes e após a facoemulsificação em olhos normais.

\section{Métodos}

Foram selecionados 221 olhos normais de 133 pacientes do Centro de Oftalmologia Avançada que foram submetidos à facoemulsificação sem intercorrências, no período de janeiro de 2006 a outubro de 2009. Utilizou-se como critérios de inclusão: 1) idade superior ou igual a 40 anos; 2) pacientes portadores de catarata senil e submetidos à facoemulsificação; 3) ausência de intercorrências trans ou pós-operatórias; 4) PIO pré-operatória menor ou igual a $21 \mathrm{mmHg} ; 5)$ miopia menor que $6 \mathrm{D}$ e hipermetropia ou astigmatismo menor que 3D; 6) ausência de doença ocular prévia, exceto a catarata; 7) ausência de cirurgia intraocular prévia; 8) capacidade física e mental para ser submetido à cirurgia. Critérios de exclusão: 1) Idade inferior a 40 anos; 2) PIO préoperatória maior que $21 \mathrm{mmHg}$; 3 ) pacientes com história de outras doenças intraoculares, que não a catarata, 4) cirurgia intraocular prévia; 5) portadores de outros tipos de catarata, que não a senil (por ex. secundárias a uveíte, trauma, uso de corticosteróides, etc.).

A dilatação pupilar no pré-operatório foi realizada com colírios de Fenilefrina 10\% e Tropicamida 1\%. Os pacientes foram submetidos a um bloqueio peribulbar com Bupivacaína $0,75 \%$ e Xilocaína 2\%, seguidos por uma gota de Iodopovidona tópico. Os pacientes utilizaram Moxifloxacino $0,5 \%$ de $3 / 3$ horas durante os cinco dias que antecederam a cirurgia.

A técnica cirúrgica utilizada foi a facoemulsificação,e todas as cirurgias foram realizadas pelo mes- mo cirurgião. Incisões em córnea clara, sendo a principal delas confeccionada com bisturi descartável de $2,75 \mathrm{~mm}$ na posição de $11 \mathrm{~h}$, e a incisão auxiliar com bisturi $15^{\circ}$ na posição de $3 \mathrm{~h}$. Injeção de substância viscoelástica dispersiva pela incisão principal. Capsulorrexis curvilínea e contínua com cistítomo, seguida por hidrodissecção e hidrodelineação. Rotação do núcleo e facoemulsificação do mesmo (dividir e conquistar). Aspiração do cortex cristaliniano utilizando a dupla via. Incisão principal ampliada para aproximadamente $3,00 \mathrm{~mm}$. Insercão da lente intraocular (Acrysof) dentro do saco capsular. Aspiração do viscoelástico e hidratação das incisões corneanas com solução salina balanceada. Curativo oclusivo com uma gota de Moxifloxacino 0,5\%, e retirada do mesmo após 24 horas. No pós-operatório foram utilizados Moxifloxacino 0,5\% de $3 / 3$ horas por 10 dias, e Dexametasona $0,1 \%$ colírio de 3/3 horas também por 10 dias. Após 10 dias, redução gradual do colírio de Dexametasona até a interrupção do mesmo, perfazendo um total de 30 dias de uso desta medicação.

A PIO foi medida pelo tonômetro de aplanação de Goldmann no pré e 1 dia, 3 dias, 1 semana, 1 mês, 3 meses, 6 meses e 1 ano no pós-operatório. Todas as medidas da PIO foram realizadas pelo mesmo examinador.

$\mathrm{Na}$ análise estatística utilizou-se o SPSS para Windows (versão 11.5). O teste não-paramétrico de Wilcoxon foi utilizado para comparar as medidas da PIO antes e 1 dia, 3 dias, 1 semana e 1 mês após a cirurgia. Nos períodos subsequentes ( 3 meses, 6 meses e 1 ano) utilizou-se o teste t-pareado.

\section{Resultados}

A idade média dos pacientes foi de 72,14 anos \pm 8,671. Foram incluídos 221 olhos de 133 pacientes, 89 $(66,9 \%)$ do sexo feminino e $44(33,1 \%)$ do sexo masculino (Tabela 1). Todos os olhos apresentavam PIO menor ou igual a $21 \mathrm{mmHg}$, e discos ópticos sem qualquer indício de glaucoma.

A tabela 2 mostra os valores descritivos para as medidas da PIO nos diferentes tempos. Pode-se observar que após 1 dia, a PIO apresentou um aumento no valor médio em relação ao pré-operatório, enquanto nos demais períodos avaliados houve uma diminuição da mesma, atingindo valor mínimo após 3 meses (Figura 1).

Como as medidas da PIO até 1 mês não apresentaram distribuição Normal, foi utilizado o teste não paramétrico de Wilcoxon. Observou-se diferença estatística entre a PIO incial e os valores após 1 dia, 3 dias, 1 


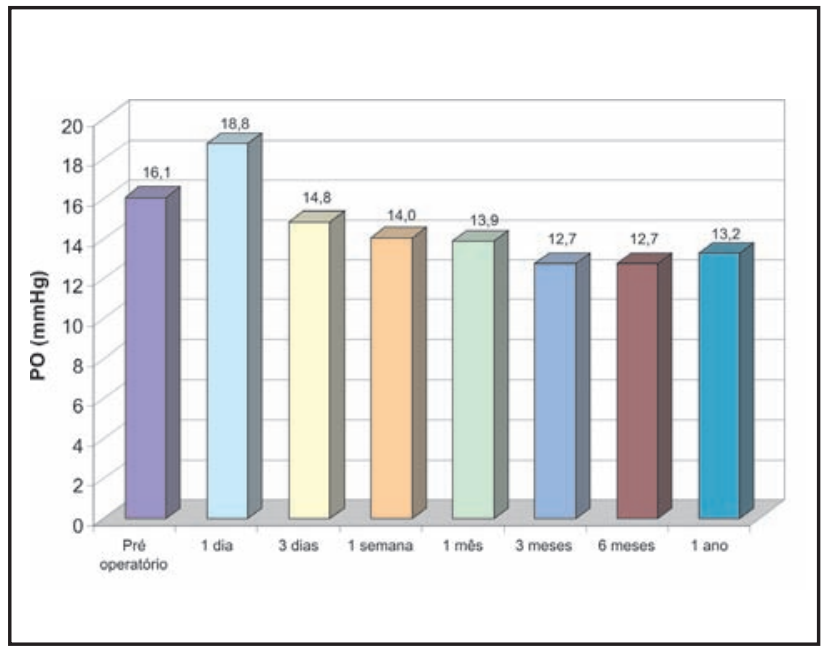

Figura 1: Valores médios das pressões no pós-operatório semana e 1 mês (valores $p<0,05$ ). Somente as pressões medidas após 3 meses, 6 meses e 1 ano apresentaram valores cuja distribuição foi Normal (Gaussiana). Desta forma, para comparação desses valores com aqueles obtidos no pré-operatório utilizou-se o teste t-pareado. Observou-se também diferença estatística entre o valor inicial e os demais valores encontrados no pós-operatório (valor $\mathrm{p}=0,00$ ).

A tabela 3 mostra a variação da PIO após a facoemulsificação. Observa-se um aumento médio da PIO após 1 dia $(-2,72 \mathrm{mmHg} \pm 5,483)$, e uma diminuição progressiva da mesma após 3 dias, atingindo seu valor mínimo após 6 meses $(3,43 \mathrm{mmHg} \pm 2,275)$, que corresponde a uma diminuição de $20,1 \%$ em relação à PIO média inicial. A queda pressórica foi mais acentuada naqueles pacientes que apresentavam PIO mais elevada no pré-operatório, conforme mostrado na figura 2 .

\section{DiscussÃo}

Estudos demonstraram mudanças no $\mathrm{ACD}^{(1-6)} \mathrm{e}$ na PIO ${ }^{(4-10)}$ após a facectomia e implante da lente intraocular.Embora existam hipóteses, ainda não se sabe o verdadeiro efeito da remoção do cristalino na PIO. Alguns autores avaliaram 56 pacientes submetidos à facoemulsificação e observaram uma diminuição da PIO a partir de 1 semana no pós-operatório, chegando a um valor mínimo com 3 meses ${ }^{(4)}$. Outros, ao estudarem prospectivamente 53 olhos de 49 pacientes submetidos à facoemulsificação, evidenciaram uma diminuição da PIO já no primeiro dia após a cirurgia. A queda pressórica foi mais acentuada naqueles pacientes que apresentavam PIO mais elevada no pré-operatório, e

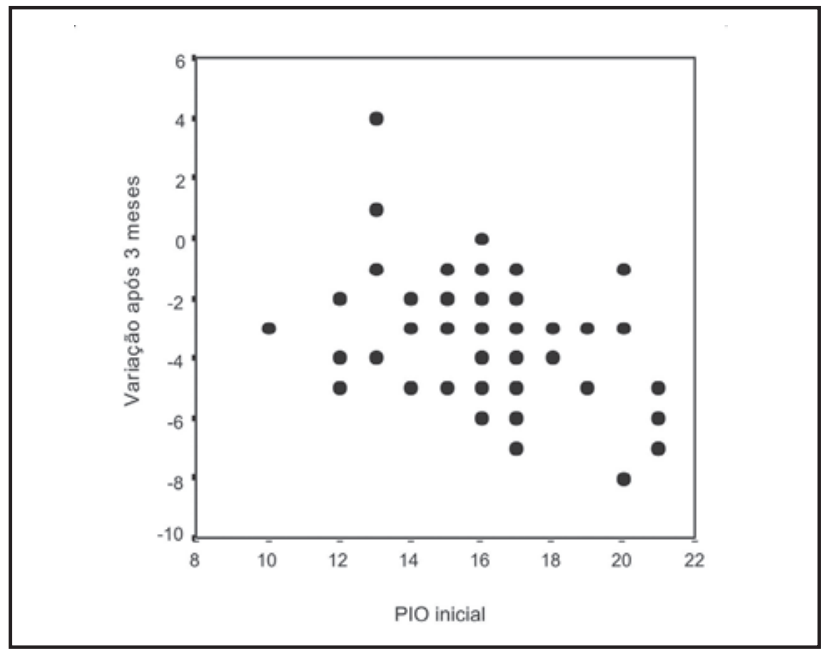

Figura 2: Variação da PIO após 3 meses

não houve diferença estatística entre os valores encontrados no pós-operatório(5). Outro autor fez uma análise comparativa entre o diâmetro axial ocular (AL) e a PIO em 71 olhos de 71 pacientes submetidos à facoemulsificação, e observou uma diminuição da PIO em olhos com menor AL $(\mathrm{AL}<25 \mathrm{~mm})^{(6)}$. Em um estudo mais recente, foram avaliados 266 pacientes normais submetidos à facoemulsificação sem intercorrências. Houve um aumento da PIO após 1 dia, e diminuição estatisticamente significativa da mesma após 3 meses (7). Já no estudo em que foram incluídos 100 olhos de 100 pacientes normais submetidos à facoemulsificação, observou-se um aumento significativo da PIO, 2 horas após a cirurgia. A PIO diminui após 24 horas e após 1 semana, chegando a valores semelhantes àqueles encontrados no pré-operatório ${ }^{(8)}$. Um outro trabalho demonstrou um aumento da PIO tanto 4 a 6 horas, quanto 24 horas após a facoemulsificação ${ }^{(9)}$. Shingleton et al. ao avaliar pacientes normais, glaucomatosos e suspeitos de glaucoma, evidenciou um aumento da PIO em todos os grupos após 24 horas de cirurgia, e uma diminuição da mesma a partir de 3 semanas, atingindo um valor mínimo após 1 ano. Em todos os intervalos, a PIO se mostrou mais alta nos grupos dos glaucomatosos e suspeitos de glaucoma ${ }^{(10)}$.

Aumento do ACD e da amplitude do seio camerular, diminuindo a resistência de escoamento do humor aquoso, podem explicar a diminuição da PIO após a facectomia ${ }^{(4)}$. Dentre outras hipóteses, estão também a liberação da prostaglandina F2 com aumento do fluxo uveoescleral, e a diminuição da produção do humor aquoso secundária à tração do corpo ciliar por fibrose e contração capsular ${ }^{(11)}$. Alguns autores demonstraram, também, que incisões menos extensas levam a 
Tabela 1

Variáveis demográficas $(n=133)$

\begin{tabular}{lcc}
\hline Variável & Média \pm DP & Percentual (\%) \\
\hline Idade & $72,14 \pm 8,671$ & - \\
Sexo & - & 66,9 \\
$\quad$ Feminino & - & 33,1 \\
$\quad$ Masculino & & \\
\hline
\end{tabular}

$\mathrm{n}=$ número de pacientes

Tabela 2

Medidas da PIO (mmHg) nos diferentes períodos

\begin{tabular}{|c|c|c|c|c|c|c|c|c|}
\hline & \multirow{2}{*}{$\begin{array}{c}\text { Pré } \\
\text { operatório }\end{array}$} & \multicolumn{7}{|c|}{ Pós operatório } \\
\hline & & 1 dia & 3 dias & 1 semana & 1 mês & 3 meses & 6 meses & 1 ano \\
\hline $\mathrm{N}$ & 221 & 217 & 34 & 148 & 112 & 54 & 42 & 37 \\
\hline Média \pm DP & $16,06 \pm 2,69$ & $18,76 \pm 5,96$ & $14,82 \pm 4,08$ & $14,03 \pm 3,06$ & $13,88 \pm 3,26$ & $12,74 \pm 2,45$ & $12,74 \pm 2,01$ & $13,24 \pm 2,77$ \\
\hline Mediana & 16,00 & 18,00 & 15,00 & 14,00 & 13,00 & 13,00 & 13,00 & 13,00 \\
\hline $\begin{array}{l}\text { Amplitude } \\
\text { (Mín - Máx) }\end{array}$ & $(10-21)$ & $(7-42)$ & $(8-28)$ & $(8-26)$ & $(7-26)$ & $(7-19)$ & $(8-18)$ & $(8-19)$ \\
\hline
\end{tabular}

$\mathrm{N}=$ número de olhos

Tabela 3

Variação da PIO (mmHg) após a facoemulsificação

\begin{tabular}{|c|c|c|c|c|c|c|c|}
\hline & 1 dia & 3 dias & 1 semana & 1 mês & 3 meses & 6 meses & 1 ano \\
\hline $\mathrm{N}$ & 217 & 34 & 148 & 112 & 54 & 42 & 37 \\
\hline Média & $-2,72$ & 1,29 & 2,24 & 2,05 & 3,39 & 3,43 & 2,95 \\
\hline$\%$ & $+18,9$ & $-6,9$ & $-12,3$ & $-12,5$ & $-19,3$ & $-20,1$ & $-19,9$ \\
\hline Desvio padrão & 5,483 & 3,762 & 2,839 & 3,122 & 2,244 & 2,275 & 2,147 \\
\hline Mínimo & -24 & -11 & -8 & -10 & -4 & 0 & -2 \\
\hline Máximo & 8 & 6 & 9 & 8 & 8 & 10 & 6 \\
\hline Percentil 25 & $-5,00$ & $-1,00$ & 1,00 & 1,00 & 2,00 & 2,00 & 1,50 \\
\hline Mediana & $-2,00$ & 2,00 & 2,00 & 3,00 & 3,00 & 3,00 & 3,00 \\
\hline Percentil 75 & 1,00 & 4,00 & 4,00 & 4,00 & 5,00 & 4,00 & 5,00 \\
\hline
\end{tabular}

$\mathrm{N}=$ número de olhos

PIO mais baixa no pós-operatório (12). Outros correlacionaram a espessura cristaliniana com a redução da PIO, de maneira que, quanto mais espesso o cristalino maior é a redução da PIO após a cirurgia (7). Já o aumento da PIO logo após a facoemulsificação, pode ser explicado por retenção de substância viscoelástica na câmara anterior, técnicas de sutura, reação inflamatória secundária a restos corticais, anestesia retrobulbar e bloqueio pupilar ${ }^{(10,13,14)}$. Embora este aumento da PIO seja frequentemente transitório, para muitos é de fundamental importância o reconhecimento de picos de pressão no pós-operatório inicial, inclusive com medi- das da PIO antes de 24 horas. Estes autores preconizam o uso de hipotensores oculares no sentido de tratar tais elevações pressóricas e evitar eventuais danos ao nervo óptico ${ }^{(8,15,16)}$.

No presente estudo, houve um aumento da PIO no primeiro dia do pós-operatório, e redução gradual da mesma a partir de 3 dias, atingindo um valor mínimo com 3 meses. A PIO diminuiu cerca de $6,9 \%$ já no terceiro dia do pós-operatório, e 19,3\%, 3 meses após a cirurgia. Pacientes que apresentavam PIO mais elevada no pré-operatório, apresentaram maior redução da mesma no pós-operatório. 


\section{Conclusão}

A cirurgia de facoemulsificação sem intercorrências, com implante de lente intraocular Acrysof dentro do saco capsular, leva a uma redução significativa da PIO em olhos normais. Houve um aumento da PIO apenas no primeiro dia do pós-operatório. A queda pressórica foi mais acentuada naqueles pacientes que apresentavam PIO mais alta no pré-operatório.

\section{Abstract}

Purpose: To assess intraocular pressure (IOP) before and after 1 day, 3 days, 1 week, 1 month, 3 months, 6 months and 1 year in normal eyes submitted to a phacoemulsification. Methods: Intraocular pressure retrospective analyses (measured by Goldmann applanation tonometer) of 221 normal eyes of 133 patients submitted to an uneventful phacoemulsification. The IOP was evaluated before and after phacoemulsification (1day, 3 days, 1 week, 1 month, 3 months, 6 months and 1 year), and these values were submitted to a comparative analyses. Results: The mean preoperative IOP of 16,06 $\pm 2,69 \mathrm{mmHg}$ rose postoperatively to $18,76 \pm 5,96 \mathrm{mmHg}$ at 1 day; dropped to $14,82 \pm 4,08$ $\mathrm{mmHg}$ at 3 days; $14,03 \pm 3,06 \mathrm{mmHg}$ at 1 week; $13,88 \pm 3,26$ mmHg at 1 month; 12,74 2 2,45 mmHg at 3 months; $12,74 \pm 2,01 \mathrm{mmHg}$ at 6 months; and 13,24 $2,77 \mathrm{mmHg}$ at 1 year. The $I O P$ rise was $18,9 \%$ at 1 day; fall of $6,9 \%$ at 3 days; $12,3 \%$ at 1 week; $12,5 \%$ at 1 month; $19,3 \%$ at 3 months; $20,1 \%$ at 6 months; and 19,9\% at 1 year. Conclusion: In normal eyes, uneventful phacoemulsification reduced IOP. The greatest IOP decreases were in patients with a higher preoperative IOP.

Keywords: Intraocular pressure; Ocular tonometry; Phacoemulsification; Lens implatation, intraocular; Lens capsule, crystalline

\section{REFERÊNCIAS}

1. Olsen T, Olesen H, Thim K, Corydon L. Prediction of postoperative intraocular lens chamber depth. J Cataract Refract Surg. 1990;16(5):587-90.

2. Naeser K, Boberg-Ans J, Bargum R. Prediction of pseudophakic anterior chamber depth from pre-operative data. Acta Ophthalmol (Copenh). 1988;66(4):433-7.

3. Yoshida S, Hashiba H, Tsukuda M, Ohara Y. Significance of angle of intraocular lens haptics on anterior chamber depth. Jpn J Clin Ophthalmol. 1989;43:173-6.
4. Cekic O, Batman C, Totan Y, Emre MI, Zilelioglu O. Changes in anterior chamber depth and intraocular pressure after phacoemulsification and posterior chamber intraocular lens implantation. Ophthalmic Surg Lasers. 1998;29(8):639-42.

5. Altan C, Bayraktar S, Altan T, Eren H, Yilmaz OF. Anterior chamber depth, iridocorneal angle width, and intraocular pressure changes after uneventful phacoemulsification in eyes without glaucoma and with open iridocorneal angles. J Cataract Refract Surg. 2004;30(4):832-8.

6. Cho YK. Early intraocular pressure and anterior chamber depth changes after phacoemulsification and intraocular lens implantation in nonglaucomatous eyes. Comparison of groups stratified by axial length. J Cataract Refract Surg. 2008;34(7):1104-9

7. Irak-Dersu I, Nilson C, Zabriskie N, Durcan J, Spencer HJ Crandall A. Intraocular pressure change after temporal clear corneal phacoemulsification in normal eyes. Acta Ophthalmol. 2010;88(1):131-4.

8. Thirumalai B, Baranyovits PR. Intraocular pressure changes and the implications on patient review after phacoemulsification. J Cataract Refract Surg. 2003;29(3):504-7.

9. Tranos PG, Wickremasinghe SS, Hildebrand D, Asaria R, Mearza A, Ghazi-Nouri S, Little BC. Same-day versus first-day review of intraocular pressure after uneventful pahcoemulsification. J Cataract Refract Surg. 2003;29(3):508-12.

10. Shingleton BJ, Gamell LS, O'Donoghue MW, Baylus SL, King $\mathrm{R}$. Long-term changes in intraocular pressure after clear corneal phacoemulsification: normal patients versus glaucoma suspect and glaucoma patients. J Cataract Refract Surg. 1999;25(7):885-90. Comment in: J Cataract Refract Surg. 1999;25(12):1560. J Cataract Refract Surg. 1999;25(7):877.

11. Cekiç O, Batman C. Hyposecretion of aqueous: another mechanism for reduced intraocular pressure after phaecoemulsification. J Cataract Refract Surg. 1998;24(5):574. Comment on: J Cataract Refract Surg. 1997;23(8):1260-4.

12. Kooner KS, Dulaney DD, Zimmerman TJ. Intraocular pressure following ECCE and IOL implantation in patients with glaucoma. Ophthalmic Surg. 1988;19(8):570-5.

13. Meyer MA, Savitt ML, Kopitas E. The effect of phacoemulsification on aqueous outflow facility. Ophthalmology. 1997;104(8):1221-7.

14. Shingleton BJ, Wadhwani RA, O'Donoghue MW, Baylus S, Hoey $\mathrm{H}$. Evaluation of intraocular pressure in the immediate period after phacoemulsification. J Cataract Refract Surg. 2001;27(4):524-7.

15. Dinakaran S, Desai SP, Raj PS. Is the first post-operative day review necessary following uncomplicated phacoemulsification surgery? Eye (Lond). 2000;14(Pt 3A):364-6.

16. Cohen VM, Demetria H, Jordan K, Lamb RJ, Vivian AJ. First day post-operative review following uncomplicated phacoemulsification. Eye (Lond). 1998;12(Pt 4):634-6.

\section{Endereço para correspondência: Nikias Alves da Silva \\ Rua Júlio Vidal, $n^{\circ} 11$ - apto 1201- Anchieta \\ CEP 30310-440 - Belo Horizonte (MG), Brasil}

E-mail: nikiasa@hotmail.com 